\title{
Light-component spectrum of the primary cosmic rays in the multi-TeV region measured by the ARGO-YBJ experiment
}

\section{B. Bartoli, ${ }^{1,2}$ P. Bernardini,${ }^{3,4}$ X. J. Bi, ${ }^{5}$ C. Bleve, ${ }^{3,6}$ I. Bolognino, ${ }^{7,8}$ P. Branchini, ${ }^{9}$ A. Budano, ${ }^{9}$} A. K. Calabrese Melcarne, ${ }^{10}$ P. Camarri, ${ }^{11,12}$ Z. Cao, ${ }^{5}$ R. Cardarelli, ${ }^{12}$ S. Catalanotti,,${ }^{1,2}$ C. Cattaneo, ${ }^{8}$ S. Z. Chen, ${ }^{5}$ T. L. Chen, ${ }^{13}$ Y. Chen,${ }^{5}$ P. Creti, ${ }^{4}$ S. W. Cui, ${ }^{14}$ B. Z. Dai, ${ }^{15}$ G. D'Alí Staiti, ${ }^{16,17}$ Danzengluobu, ${ }^{13}$ M. Dattoli, ${ }^{18,19,20}$ I. De Mitri, ${ }^{3,4}$ B. D'Ettorre Piazzoli, ${ }^{1,2}$ T. Di Girolamo, ${ }^{1,2}$ X. H. Ding ${ }^{13}$ G. Di Sciascio, ${ }^{12}$ C. F. Feng, ${ }^{21}$ Zhaoyang Feng, ${ }^{13}$ Zhenyong Feng, ${ }^{22}$ E. Giroletti, ${ }^{7,8}$ Q. B. Gou, ${ }^{5}$ Y. Q. Guo, ${ }^{5}$ H. H. He, ${ }^{5}$ Haibing Hu, ${ }^{13}$ Hongbo Hu, ${ }^{5}$ Q. Huang, ${ }^{22}$ M. Iacovacci, ${ }^{1,2}$ R. Iuppa, ${ }^{11,12}$ I. James,${ }^{23,9}$ H. Y. Jia, ${ }^{22}$ Labaciren, ${ }^{13}$ H. J. Li,${ }^{13}$ J. Y. Li ${ }^{21}$ X. X. Li, ${ }^{5}$ G. Liguori, ${ }^{7,8}$ C. Liu, ${ }^{5}$ C. Q. Liu, ${ }^{15}$ J. Liu, ${ }^{15}$ M. Y. Liu, ${ }^{13}$ H. Lu, ${ }^{5}$ X. H. Ma, ${ }^{5}$ G. Mancarella, ${ }^{3,4}$ S. M. Mari, ${ }^{23,9, *}$ G. Marsella, ${ }^{4,24}$ D. Martello, ${ }^{3,4}$ S. Mastroianni, ${ }^{2}$ P. Montini, ${ }^{23,9}$ C. C. Ning, ${ }^{13}$ A. Pagliaro, ${ }^{17,25}$ M. Panareo, ${ }^{4,24}$ B. Panico, ${ }^{11,12}$ L. Perrone,${ }^{4,24}$ P. Pistilli, ${ }^{23,9}$ X. B. Qu ${ }^{21}$ F. Ruggieri, ${ }^{9}$ P. Salvini, ${ }^{8}$ R. Santonico, ${ }^{11,12}$ P. R. Shen, ${ }^{5}$ X. D. Sheng, ${ }^{5}$ F. Shi,${ }^{5}$ C. Stanescu, ${ }^{9}$ A. Surdo, ${ }^{4}$ Y. H. Tan, ${ }^{5}$ P. Vallania, ${ }^{18,19}$ S. Vernetto, ${ }^{18,19}$ C. Vigorito, ${ }^{19,20}$ B. Wang, ${ }^{5}$ H. Wang, ${ }^{5}$ C. Y. Wu, ${ }^{5}$ H. R. Wu,${ }^{5}$ B. Xu, ${ }^{22}$ L. Xue, ${ }^{21}$ X.X. Yan, ${ }^{15}$ Q. Y. Yang, ${ }^{15}$ X. C. Yang,${ }_{1}^{15}$ Z. G. Yao, ${ }^{5}$ A. F. Yuan, ${ }^{13}$ M. Zha, ${ }^{5}$ H. M. Zhang,,${ }^{5}$ Jilong Zhang, ${ }^{5}$ Jianli Zhang, ${ }^{5}$ L. Zhang, ${ }^{15}$ P. Zhang, ${ }^{15}$ X. Y. Zhang, ${ }^{21}$ Y. Zhang, ${ }^{5}$ Zhaxiciren, ${ }^{13}$ Zhaxisangzhu, ${ }^{13}$ X. X. Zhou, ${ }^{22}$ F. R. Zhu, ${ }^{22}$ Q. Q. Zhu, ${ }^{5}$ and G. Zizzi ${ }^{10}$

\section{(ARGO-YBJ Collaboration)}

${ }^{1}$ Dipartimento di Fisica dell'Università di Napoli “Federico II,” Complesso Universitario di Monte Sant'Angelo, via Cinthia, 80126 Napoli, Italy

${ }^{2}$ Istituto Nazionale di Fisica Nucleare, Sezione di Napoli, Complesso Universitario di Monte Sant'Angelo, via Cinthia, 80126 Napoli, Italy

${ }^{3}$ Dipartimento di Fisica dell'Università del Salento, via per Arnesano, 73100 Lecce, Italy

${ }^{4}$ Istituto Nazionale di Fisica Nucleare, Sezione di Lecce, via per Arnesano, 73100 Lecce, Italy

${ }^{5}$ Key Laboratory of Particle Astrophysics, Institute of High Energy Physics, Chinese Academy of Sciences, Post Office Box 918,100049 Beijing, People's Republic of China

${ }^{6}$ Istituto Nazionale di Fisica Nucleare, Sezione di Lecce, via per Arnesano, 73100 Lecce, Italy

${ }^{7}$ Dipartimento di Fisica Nucleare e Teorica dell'Università di Pavia, via Bassi 6, 27100 Pavia, Italy

${ }^{8}$ Istituto Nazionale di Fisica Nucleare, Sezione di Pavia, via Bassi 6, 27100 Pavia, Italy

${ }^{9}$ Istituto Nazionale di Fisica Nucleare, Sezione di Roma Tre, via della Vasca Navale 84, 00146 Roma, Italy

${ }^{10}$ Istituto Nazionale di Fisica Nucleare-CNAF, Viale Berti-Pichat 6/2, 40127 Bologna, Italy

${ }^{11}$ Dipartimento di Fisica dell'Università di Roma "Tor Vergata," via della Ricerca Scientifica 1, O0133 Roma, Italy

${ }^{12}$ Istituto Nazionale di Fisica Nucleare, Sezione di Roma Tor Vergata, via della Ricerca Scientifica 1, 00133 Roma, Italy

${ }^{13}$ Tibet University, 850000 Lhasa, Xizang, People's Republic of China

${ }^{14}$ Hebei Normal University, Shijiazhuang 050016, Hebei, People's Republic of China

${ }^{15}$ Yunnan University, 2 North Cuihu Road, 650091 Kunming, Yunnan, People's Republic of China

${ }^{16}$ Università degli Studi di Palermo, Dipartimento di Fisica e Tecnologie Relative, Viale delle Scienze, Edificio 18, 90128 Palermo, Italy

${ }^{17}$ Istituto Nazionale di Fisica Nucleare, Sezione di Catania, Viale Andrea Doria 6, 95125 Catania, Italy

${ }^{18}$ Istituto di Fisica dello Spazio Interplanetario dell'Istituto Nazionale di Astrofisica, corso Fiume 4, 10133 Torino, Italy

${ }^{19}$ Istituto Nazionale di Fisica Nucleare, Sezione di Torino, via Pietro Giuria 1, 10125 Torino, Italy

${ }^{20}$ Dipartimento di Fisica Generale dell'Università di Torino, via Pietro Giuria 1, 10125 Torino, Italy

${ }^{21}$ Shandong University, 250100 Jinan, Shandong, People's Republic of China

${ }^{22}$ Southwest Jiaotong University, 610031 Chengdu, Sichuan, People's Republic of China

${ }^{23}$ Dipartimento di Fisica dell'Università "Roma Tre," via della Vasca Navale 84, 00146 Roma, Italy

${ }^{24}$ Dipartimento di Ingegneria dell'Innovazione, Università del Salento, 73100 Lecce, Italy

${ }^{25}$ Istituto di Astrofisica Spaziale e Fisica Cosmica dell'Istituto Nazionale di Astrofisica, via La Malfa 153, 90146 Palermo, Italy (Received 7 November 2011; published 9 May 2012)

The ARGO-YBJ experiment detects extensive air showers in a wide energy range by means of a full-coverage detector which is in stable data taking in its full configuration since November 2007 at the YBJ International Cosmic Ray Observatory (4300 m a.s.l., Tibet, People's Republic of China). In this paper the measurement of the light-component spectrum of primary cosmic rays in the energy region

\footnotetext{
*Corresponding author.
} mari@fis.uniroma3.it 
$(5 \div 200) \mathrm{TeV}$ is reported. The method exploited to analyze the experimental data is based on a Bayesian procedure. The measured intensities of the light component are consistent with the recent CREAM results and higher than that obtained adding the proton and helium spectra reported by the RUNJOB experiment.

\section{INTRODUCTION}

The cosmic ray spectrum spans a huge energy interval up to $10^{20} \mathrm{eV}$ or more [1,2]. Many experimental efforts have been devoted to the study of cosmic ray properties. However, despite a huge amount of experimental data the site of their origin and the mechanisms of acceleration and propagation are still under discussion. The observation techniques can be grouped into two broad classes: direct and indirect measurements. Direct measurements can access only the low-energy region, up to a few hundred $\mathrm{TeV}$, because of the restricted dimensions and the limited exposure of the detectors. The measurement technique based on the observation of extensive air showers (EASs) with surface arrays allows the detection of events induced by primary particles with energies up to $10^{20} \mathrm{eV}$. Unless complementary techniques provide an independent calibration, the measurement of the energy spectrum relies on Monte Carlo simulations in order to unfold the relevant information from collected data. The indirect measurements in the region of a hundred $\mathrm{TeV}$ show an overall agreement between the experiments within a factor of 2 [3], while the balloon-borne experiments have to cope with the systematic uncertainties due to the analysis of data taken during different flights. Recent more precise measurements carried out by the long duration flights of the balloon-borne CREAM experiment [4,5] show that the proton and helium spectra from 2.5 to $250 \mathrm{TeV}$ are both flatter compared with the lower energy measurements. In particular, the proton spectrum in this energy range is found harder than the value quoted in [3] and obtained by fitting many previous direct measurements. In addition, the proton and helium fluxes measured by CREAM are consistent with the measurements of JACEE [6] and higher, particularly for helium, in comparison with RUNJOB [7]. The evolution of the proton and helium spectra and their subtle differences can be an indication of a different population of cosmic ray sources or acceleration sites. In many current models, as, for instance, those proposed in $[8,9]$, a scenario is envisaged, in which the explosion of normal supernovae directly into the interstellar medium and the explosion of massive stars into their former stellar wind, like that of Wolf-Rayet stars, are the main sources of cosmic rays up to the knee region. If elements heavier than hydrogen come mainly from the latter sources, their spectra are expected to be harder than the proton spectrum and extending to higher energies. Since proton and helium nuclei are the bulk of the cosmic rays at energies below the knee $\left(\sim 3 \times 10^{15} \mathrm{eV}\right)$, the study of their spectrum in this energy region is of primary importance. A better understanding of this topic can be obtained by extending the indirect measurements to the low-energy region covered by balloons and satellite detectors. The ARGO-YBJ experiment [10-12], characterized by low-energy threshold and high duty cycle, is able to overlap direct measurements in a wide region below $100 \mathrm{TeV}$, not accessible by other EAS experiments. In this paper we evaluate the light-component spectrum of the primary cosmic rays in the energy region $(5 \div$ 200) $\mathrm{TeV}$ by using a Bayesian approach. In Sec. II the main characteristics of the detector are briefly described. In Sec. III the Bayesian method used for this analysis, previously presented in [13], is outlined. In Sec. IV the Monte Carlo events used in the Bayesian unfolding procedure are described. Section V is devoted to the data analysis, and the results are presented in Sec. VI and discussed in Sec. VII. Section VIII summarizes the main conclusions of the work.

\section{THE ARGO-YBJ DETECTOR}

ARGO-YBJ is a "full-coverage" air shower array operating at the Yangbajing International Cosmic Ray Observatory, located in the Tibet region (People's Republic of China) at an altitude of $4300 \mathrm{~m}$ above sea level. One of the main physics items of the experiment is the study of the cosmic rays in the $(1 \div 10000) \mathrm{TeV}$ energy range. The detector consists of a single layer of resistive plate chambers (RPCs) [12] covering an area of $74 \times 78 \mathrm{~m}^{2}$. The basic module of the detector is the cluster $\left(5.7 \times 7.6 \mathrm{~m}^{2}\right)$ made of 12 RPCs $\left(2.85 \times 1.23 \mathrm{~m}^{2}\right.$ each $)$. One hundred and thirty clusters are assembled in a fullcoverage carpet of $5800 \mathrm{~m}^{2}$ with an active area of about 93\%. This carpet is surrounded by 23 additional clusters, arranged in a guard ring with a coverage of about $40 \%$ to improve the reconstruction of the core position and the angular resolution. The total area of the array is $110 \times$ $100 \mathrm{~m}^{2}$. Each RPC is read out via 80 strips $(6.75 \times$ $\left.61.80 \mathrm{~cm}^{2}\right)$, logically organized in 10 pads $(55.6 \times$ $61.8 \mathrm{~cm}^{2}$ ). Each strip represents the space granularity of the detector, i.e., the pixel used to sample the particles of the shower front. Each pad signal, which is realized by 8 strips, is sent to a time-to-digital converter and represents the time pixel, allowing a resolution of about $1.8 \mathrm{~ns}$ in measuring the particle arrival time [14]. In order to extend the measurable primary energy range up to the $\mathrm{PeV}$ region, each RPC is also equipped with 2 large pads $(139 \times$ $123 \mathrm{~cm}^{2}$ ) with analog readout [15]. The whole system allows a detailed reconstruction of the shower front with a high space-time resolution. The detector performance is 
discussed in $[12,16]$. The angular resolution has been estimated using different methods and is better than $0.6^{\circ}$ [17] for events with more than 200 fired pads. The installation of the central carpet was completed in June 2006. The guard ring was completed during spring 2007 and was plugged in the data acquisition system [18] in November 2007. The data analyzed in this paper have been collected during the first months of 2008.

\section{UNFOLDING THE PRIMARY SPECTRUM BY USING THE BAYESIAN METHOD}

As it is well-known, the development of atmospheric showers exhibits large fluctuations. For this reason, the energy distribution of the incoming particles cannot be obtained on an event-by-event basis, but must be evaluated by means of an unfolding procedure. The observable measured by ARGO-YBJ is the multiplicity distribution $N(M)$, which represents the number of events with a given number $M$ of fired strips (multiplicity), collected in a fixed period $\Delta T$ and accepted within a solid angle $\Omega$. The rate of observed events is obtained by integrating the primary cosmic ray differential intensity $N(E)$ on the energy range of the primary particles and on the overall acceptance of the detector:

$$
N(M)=\int_{E_{1}}^{E_{2}} \int_{\Omega} A_{\mathrm{eff}}\left(E^{\prime}, M, \vartheta^{\prime}\right) N(E) d \phi \sin \vartheta^{\prime} d \vartheta^{\prime} d E^{\prime},
$$

where $A_{\mathrm{eff}}(E, M, \vartheta)$ is a function of the primary particle energy $E$, of the number of fired strips $M$, and of the solid angle $\Omega$. In this paper the analysis is restricted to nearly vertical events, where the effective area depends only weakly on the zenith angle $\vartheta$, so it is averaged by considering the mean value within the whole observation solid angle:

$$
\bar{A}_{\mathrm{eff}}(E, M)=\frac{1}{\Omega} \int_{\Omega} A_{\mathrm{eff}}\left(E, M, \vartheta^{\prime}\right) d \phi \sin \left(\vartheta^{\prime}\right) d \vartheta^{\prime} .
$$

Inserting Eq. (2) in Eq. (1), the rate of measured events is given by

$$
N(M)=\Omega \int_{E_{1}}^{E_{2}} \bar{A}_{\mathrm{eff}}\left(E^{\prime}, M\right) N\left(E^{\prime}\right) d E^{\prime} .
$$

This equation represents the connection between the observable quantity $N(M)$ and the primary cosmic ray intensity $N(E)$. The left side of Eq. (3) is the rate of events measured by the ARGO-YBJ detector after applying selection criteria on measured data. Extracting the cosmic ray flux from Eq. (3) is a classical unfolding problem [19] and can be tackled by means of the Bayesian technique [20]. The classical approach is based on a fitting procedure which requires formulating hypotheses on the shape of the spectra of different primaries. On the contrary, the Bayesian unfolding method does not require any assumption about the shape of the primary spectra. The relevant quantities used in this analysis are

$P(E)$ : the probability distribution of primary cosmic rays of energy $E$, the quantity to be obtained.

$P(M)$ : the probability distribution to detect showers with multiplicity $M$, the quantity measured by the ARGO-YBJ detector.

$P(M \mid E)$ : conditioned probability, namely, the probability that a shower induced by a primary cosmic ray of energy $E$ is detected with multiplicity $M$.

These probabilities are connected by the Bayes theorem which, in the case of $n$ independent causes (energies) $E_{i}\left(i=1, \ldots, n_{E}\right)$ responsible for the effects (multiplicities) $M_{j}\left(j=1, \ldots, n_{M}\right)$, states that

$$
\begin{gathered}
P\left(E_{i}\right) \propto \sum_{j=1}^{n_{M}} P\left(E_{i} \mid M_{j}\right) \cdot P\left(M_{j}\right), \\
P\left(E_{i} \mid M_{j}\right)=\frac{P\left(M_{j} \mid E_{i}\right) \cdot P\left(E_{i}\right)}{\sum_{l=1}^{n_{E}} P\left(M_{j} \mid E_{l}\right) P\left(E_{l}\right),}
\end{gathered}
$$

where $P\left(E_{i} \mid M_{j}\right)$ and $P\left(M_{j} \mid E_{i}\right)$ are the conditioned probabilities, namely, the probabilities connecting events with energy $E_{i}$ to events with multiplicity $M_{j}$. In the Bayesian unfolding scheme, $P\left(M_{j} \mid E_{i}\right)$ must be evaluated by means of a Monte Carlo simulation, the $P\left(M_{j}\right)$ are calculated from the experimental data, and the $P\left(E_{i}\right)$ are related to the cosmic ray differential intensity. The Bayesian analysis is performed with an iterative procedure: the $P\left(E_{i} \mid M_{j}\right)$ are computed by Eq. (5) assuming a starting value of the energy distribution $P\left(E_{i}\right)$. Using Eq. (4), a more accurate value of $P\left(E_{i}\right)$ is derived and used to iterate. The iterative procedure ends when the variations on the value of $P\left(E_{i}\right)$ are negligible. The relevant quantities $P\left(E_{i}\right)$ and $P\left(M_{j}\right)$ are defined as follows:

$$
\begin{gathered}
P\left(E_{i}\right)=\frac{N\left(E_{i}\right)}{\sum_{E^{\prime}} N\left(E^{\prime}\right)}, \\
P\left(M_{j}\right)=\frac{N\left(M_{j}\right)}{N_{\mathrm{sel}}},
\end{gathered}
$$

where $N\left(E_{i}\right)$ is the number of events with energy $E_{i}, N_{\text {sel }}$ is the total number of selected events, and $N\left(M_{j}\right)$ is the number of detected events with multiplicity $M_{j}$. The $P\left(M_{j} \mid E_{i}\right)$ quantities have been computed by using a Monte Carlo simulation described in the following section.

\section{THE MONTE CARLO DATA SAMPLE}

The events have been generated by using the CORSIKA code [21] (ver. 6.710), which provides a complete simulation of the shower development in the Earth's atmosphere. The electromagnetic part of the shower simulation is implemented by means of the EGS4 code [22,23], while 
for the hadronic component several options [24] are available. The Monte Carlo events have been generated by using the QGSJET-II interaction model [25] for the highenergy hadronic interactions and by the GHEISHA interaction model [26] for the low-energy hadronic interactions. The data have been generated in the energy range $(1 \div$ 10 000) $\mathrm{TeV}$ with distribution given by

$$
N(E) d E=N_{0} E^{-\gamma} d E .
$$

A total number of about $2.27 \times 10^{7}$ events have been generated with a spectral index $\gamma=2.78$. The resulting showers have been sampled at the Yangbajing altitude. In order to accurately reproduce the detector response, a simulation based on GEANT3 [27] has been applied. The accidental background generated by each pad has been included and a full trigger simulation performed, taking into account the measured efficiency and the time resolution of RPCs. Monte Carlo events have been produced in the same format as data and processed via the same reconstruction code. Only protons, helium nuclei, and CNO nuclei have been generated extensively, because the heavier components give a minor contribution in the energy interval explored in this work $[7,28,29]$. Indeed, showers initiated by heavy nuclei develop and are absorbed faster in the atmosphere. At a given energy heavy nuclei thus produce showers of smaller size at the observation level. The contribution of the CNO component is reduced by the cuts used in this analysis (described in Sec. V) and it turns out to be less than 2\% (see Fig. 3). The Monte Carlo data set is composed of $91 \%$ protons and $9 \%$ helium nuclei according to the RUNJOB measurements [30]. The shower axis is randomly distributed over an area $A_{g}$ centered on the ARGO-YBJ detector. Different $A_{g}$ have been chosen for the different energy bins used in the simulation, ranging from $(100 \times 100) \mathrm{m}^{2}$ in the lower energy bins up to $(400 \times 400) \mathrm{m}^{2}$ in the higher energy bins. However, we

point out that in the Bayesian approach the actual value of $A_{g}$ is not relevant.

\section{DATA ANALYSIS}

The ARGO-YBJ detector takes data by using an inclusive trigger [31] requiring a number of fired pads $N_{\text {pad }} \geq$ $N_{\text {trig }}$ in the central carpet, within a time coincidence window of 420 ns. The detector was in stable data taking during the runs selected for this analysis; $N_{\text {trig }}$ was set to 20 , with a resulting trigger rate of $\sim 3.5 \mathrm{kHz}$ and a dead time of $\sim 4 \%$. In Fig. 1 the space-time view of a typical event is shown. The high segmentation of the readout allows a high granularity imaging of the space-time structure of the shower front, which is used to reconstruct the shower direction and the core position. Special care is devoted to the time calibration procedure [32] of the 18360 pads of the detector in order to get a high pointing accuracy and good angular resolution. The angular resolution improves increasing the number of fired pads. Experimental results show that the angular resolution $\psi_{72}$, defined as the opening angle containing $71.5 \%$ of the reconstructed events coming from a fixed direction, is about $0.4^{\circ}$ for events with pad multiplicity of about 500 [17]. The core position $\left(x_{\text {core }}, y_{\text {core }}\right)$ of each shower is estimated by using the likelihood method based on the Nishimura-Kamata-Greisen function [33-35]. The accuracy of the core position reconstruction is estimated to be about $5 \mathrm{~m}$ from Monte Carlo studies for events with the core hitting the detector. A first selection of the data has been based on the quality of the reconstruction procedure. Additional cuts have been applied in order to estimate with good accuracy the probabilities used in the unfolding procedure and to make negligible the contamination by external events (i.e., showers with core position outside the detector but reconstructed incorrectly inside). The

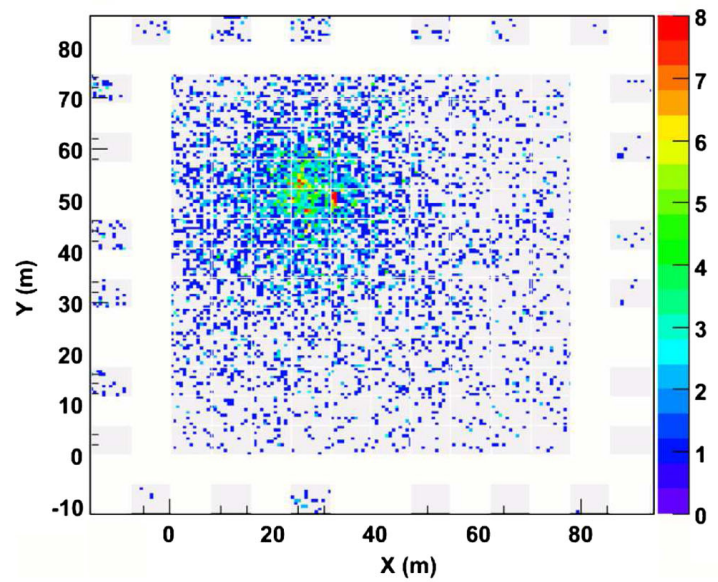

(a) $\mathrm{x}-\mathrm{y}$ projection

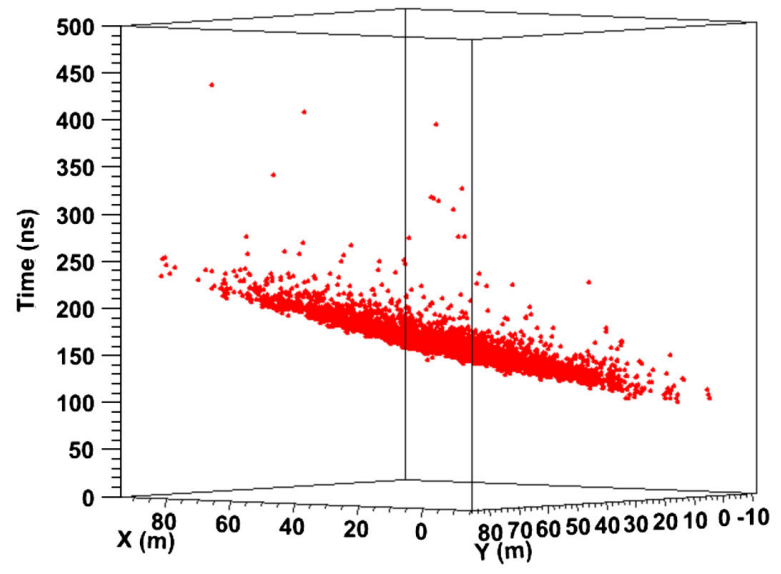

(b) $x, y, t$ view

FIG. 1 (color online). A typical event triggered by the ARGO-YBJ detector. The space hit density is obtained from the pattern in the $x-y$ projection, whereas the arrival direction is reconstructed from the time distribution, shown on the vertical coordinate. 
following selection criteria have been adopted for both Monte Carlo events and data:

(a) The Monte Carlo events used in the analysis have been generated with the zenith angle $\left(\vartheta_{G}\right)$ in the range $0^{\circ} \div 45^{\circ}$. In order to avoid bias effects in estimating the quantities $P\left(M_{j} \mid E_{i}\right)$ and to improve the quality of the reconstruction, data and Monte Carlo events have been selected requiring the reconstructed zenith angle $\left(\vartheta_{R}\right)$ in the range $0^{\circ} \div 30^{\circ}$. This cut sets the solid angle $\Omega$ to about 0.842 sr. In Fig. 2 the distribution of the reconstructed zenith angle $\vartheta_{R}$ versus the generated zenith angle $\vartheta_{G}$ is reported, and the cut on $\vartheta_{R}$ is also shown.

(b) The Monte Carlo events have been generated in the energy range $(1 \div 10000) \mathrm{TeV}$. In order to select well reconstructed events and reduce bias effects in the estimate of the Bayesian probabilities, mainly located at the edges of the energy range used in this analysis, the events have been selected requiring the strip multiplicity $M$, measured for each event, in the range $500 \leq M \leq 50000$. This cut selects events well within the energy range used for the event simulation.

(c) The rejection of events falling far from the detector is achieved by comparing the average particle density measured by the innermost 20 clusters $\left(\rho_{\text {in }}\right)$ to that measured by the outermost 42 ones $\left(\rho_{\text {out }}\right)$. By requiring $\rho_{\text {in }}>1.25 \times \rho_{\text {out }}$, the core position turns out to be inside an area of about $40 \times 40 \mathrm{~m}^{2}$ centered on the detector. No events generated outside the detector have been selected by this procedure. The fraction of events passing the cuts used in this

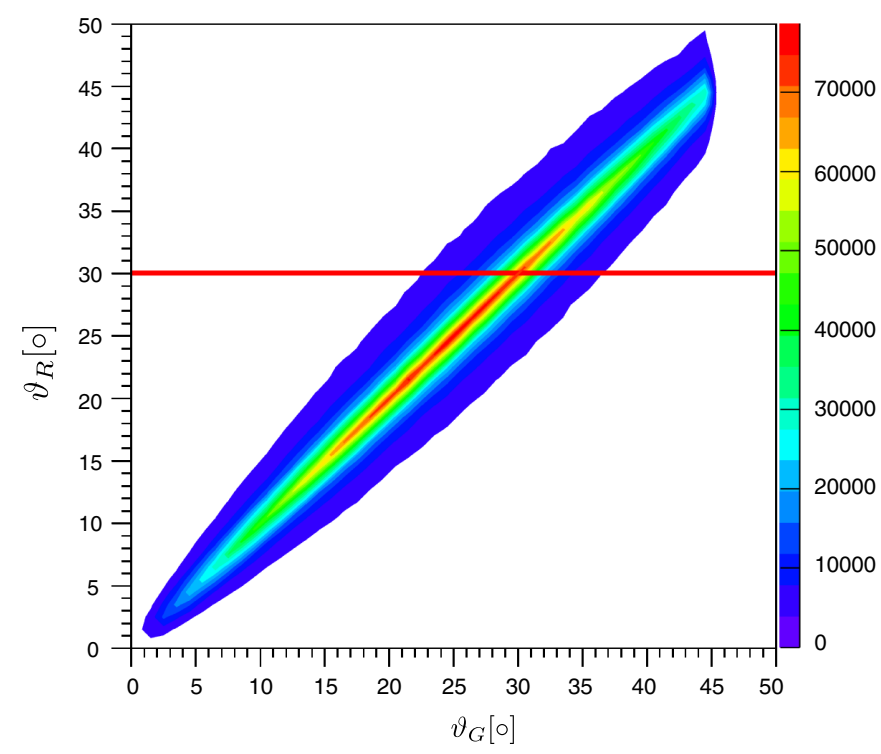

FIG. 2 (color online). The distribution of the reconstructed zenith angle $\vartheta_{R}$ versus the generated zenith angle $\vartheta_{G}$. The cut on $\vartheta_{R}$ is shown by the horizontal line. analysis has been checked to be consistent with the Monte Carlo prediction, being about $24 \%$ both in data and simulations.

Moreover, only about $2 \%$ of the showers induced by the CNO group pass the cuts used in this analysis; the cut on the particle density $\left(\rho_{\text {in }}\right)$ selects showers with well-shaped core discarding events induced by heavier primaries. The effects of the cuts are shown in Figs. 3 and 4. In Fig. 3 the fraction of the generated events selected by the cuts is shown as a function of the energy for hydrogen, helium, and CNO primary nuclei. This figure shows that the events outside the energy range $\left(10^{3} \div 10^{7}\right) \mathrm{GeV}$ give a negligible contribution to the estimation of the probabilities $P\left(M_{j} \mid E_{i}\right)$; furthermore the fraction of CNO induced showers selected by the cuts is reduced by about 10 times compared with the fraction of protons and helium nuclei. In Fig. 4 the distributions of the $X$ and $Y$ coordinates of the core are shown both for Monte Carlo events and data. The figure shows clearly that the events selected by the cuts are located in an area of about $40 \times 40 \mathrm{~m}^{2}$ centered on the detector.

\section{RESULTS}

\section{A. The unfolding procedure}

In the Bayesian method the energy bins are independent, without constraints among each other [36]. Three data samples, of about $10^{6}$ events each, have been generated with spectral index $\gamma=1,2,2.78$, in a cone of opening angle $45^{\circ}$ around the vertical direction. The Bayesian procedure has been then applied, the final results $N(E)$ turning out to be completely independent of the spectral indexes $\gamma$ used in the simulation. In order to measure the light-component spectrum, the Monte Carlo events generated with spectral index $\gamma=2.78$ have been

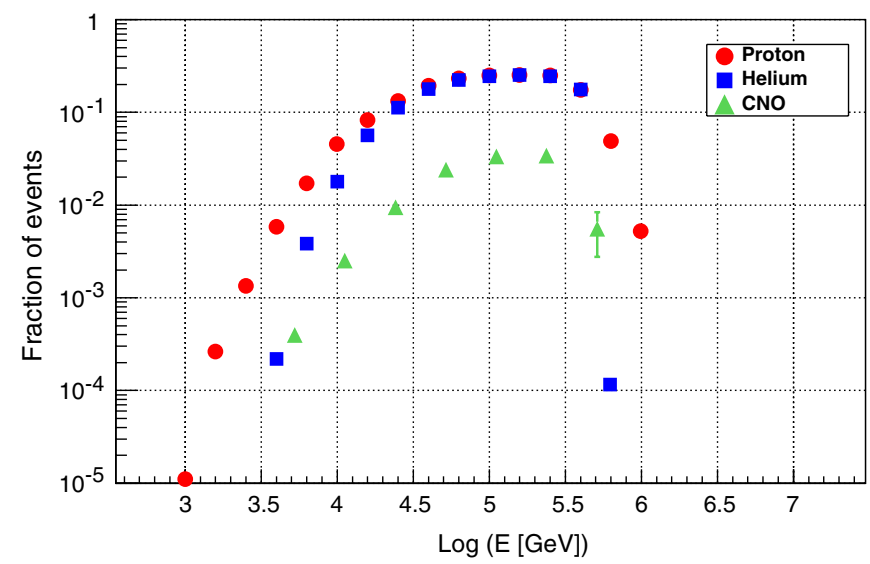

FIG. 3 (color online). The fraction of the simulated events selected by the cuts used in this analysis is shown as a function of the energy for proton induced events (red circles), helium induced events (blue squares), and CNO induced events (green triangles). 

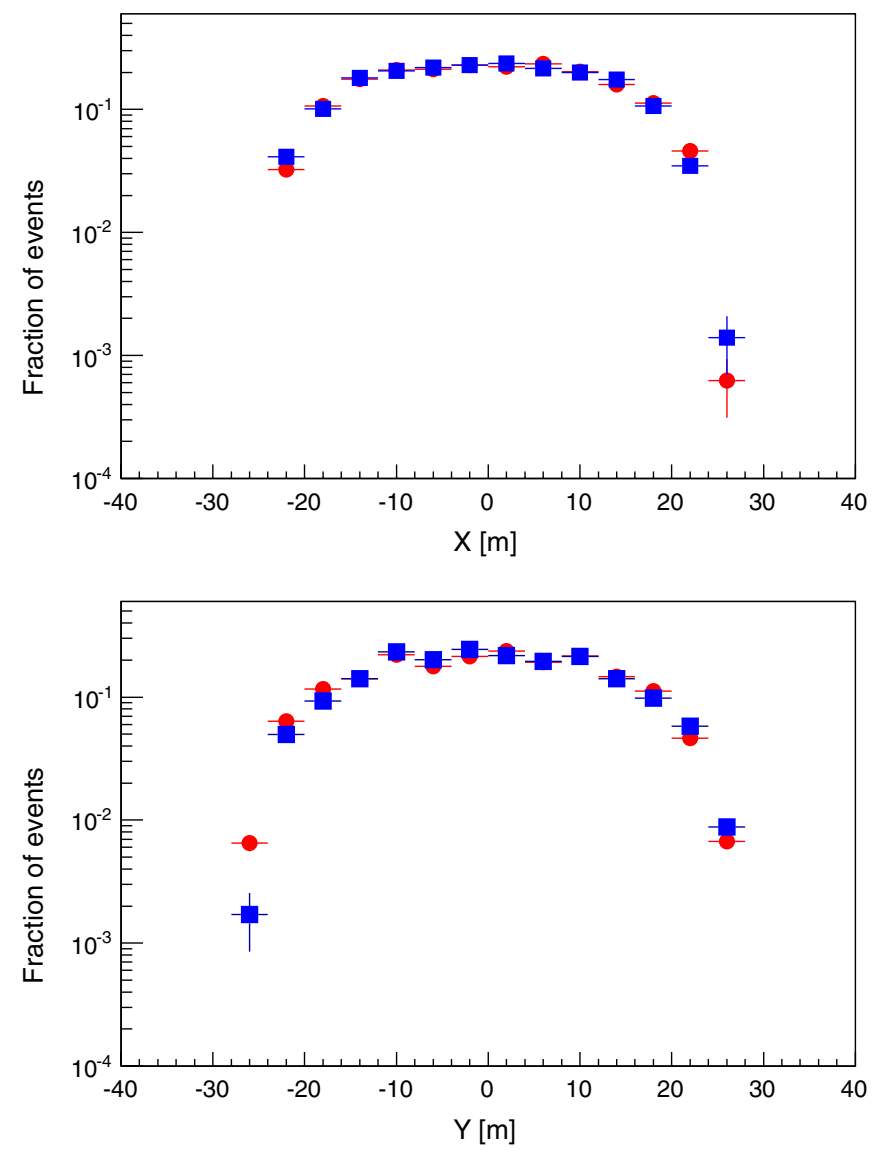

FIG. 4 (color online). Distributions of the $X$ and $Y$ coordinates of the core for Monte Carlo events (red circles) and for data (blue squares) selected by the criteria used in this analysis.

sorted in seven energy bins and 12 multiplicity bins. The same multiplicity bins have been used to analyze a sample of data (see Table I) consisting of three runs of about $25.0 \times 10^{6}$ events each, collected during the period January-May 2008. The Bayesian unfolding has been performed by using a flat distribution as the initial value of the $P\left(E_{i}\right)$. The effect of using different prior distributions $P\left(E_{i}\right)$ has been evaluated as negligible. A soft smoothing [13] has been applied to the $n$th value of $P\left(E_{i}\right)$ during the recursive procedure in order to ensure a stable convergence. A dedicated procedure has been used in order to evaluate the effective alive time of the detector for the three runs used in this analysis, which results to be about $96.2 \%$ of the data taking period. The intensities $N(E)$ of the light-component energy spectrum obtained by this analysis are reported in the last column (full sample) of Table I. The Bayesian procedure was then applied to each of the three runs separately. The results shown in Table I provide a valuation of the systematic effects, mainly due to the accuracy of the simulation of the detector response and to the seasonal effect of the shower flux (see next section).

\section{B. Evaluation of systematic uncertainties}

The measurements of the intensity of the lightcomponent energy spectrum given in Table I are affected by a statistical error of the order of $\pm 1 \%$. A study of the possible systematic effects has been performed. The sources of systematic effects considered in this work are

(i) Effects due to the selection cuts on the following measured quantities: zenith angle $\vartheta$, multiplicity $M$, and particle density $\rho$. The uncertainty on the measured spectrum, estimated by applying large variations to the selection cuts, is about $\pm 5 \%$.

(ii) Effects due to the reliability of the simulation of the detector response. The relation between the measured strip multiplicity and the primary energy distribution has been studied in [37] and has been found to be in good agreement with Monte Carlo predictions. The reliability of the simulation procedure has been checked by comparing the distributions of several variables obtained applying the same selection cuts to data and to Monte Carlo events. As an example the distribution of the core position, reported in Fig. 4, shows that the same fraction of events in the data and Monte Carlo sample has been selected in the inner detector area. Moreover, the strip multiplicity distribution obtained from the Monte Carlo events has been compared with the experimental distribution by successfully using the Kolmogorov-Smirnov test with $K=0.035$ and 30 d.o.f. (see Fig. 5).

TABLE I. Intensity of the light-component energy spectrum obtained by using a Bayesian method applied to about $75 \times 10^{6}$ events collected in year 2008 (see text for details).

\begin{tabular}{lccccc}
\hline \hline$E_{\min }-E_{\max }[\mathrm{GeV}]$ & $\begin{array}{c}\text { Mean energy } \\
{[\mathrm{GeV}]}\end{array}$ & Run 1 & N(E) \pm statistical errors $\left[\mathrm{m}^{-2} \mathrm{~s}^{-1} \mathrm{sr}^{-1} \mathrm{GeV}^{-1}\right]$ & \\
& Run 2 & Run 3 & Full sample \\
\hline $5.50 \times 10^{3}-9.55 \times 10^{3}$ & $7.35 \times 10^{3}$ & $(8.62 \pm 0.09) \times 10^{-7}$ & $(9.40 \pm 0.09) \times 10^{-7}$ & $(7.88 \pm 0.08) \times 10^{-7}$ & $(7.91 \pm 0.05) \times 10^{-7}$ \\
$9.55 \times 10^{3}-1.66 \times 10^{4}$ & $1.25 \times 10^{4}$ & $(1.75 \pm 0.02) \times 10^{-7}$ & $(1.88 \pm 0.02) \times 10^{-7}$ & $(1.81 \pm 0.02) \times 10^{-7}$ & $(1.77 \pm 0.01) \times 10^{-7}$ \\
$1.66 \times 10^{4}-2.88 \times 10^{4}$ & $2.16 \times 10^{4}$ & $(3.87 \pm 0.04) \times 10^{-8}$ & $(4.12 \pm 0.04) \times 10^{-8}$ & $(4.12 \pm 0.04) \times 10^{-8}$ & $(4.11 \pm 0.02) \times 10^{-8}$ \\
$2.88 \times 10^{4}-5.01 \times 10^{4}$ & $3.68 \times 10^{4}$ & $(1.02 \pm 0.01) \times 10^{-8}$ & $(1.07 \pm 0.01) \times 10^{-8}$ & $(1.07 \pm 0.01) \times 10^{-8}$ & $(1.03 \pm 0.01) \times 10^{-8}$ \\
$5.01 \times 10^{4}-8.91 \times 10^{4}$ & $6.40 \times 10^{4}$ & $(2.44 \pm 0.02) \times 10^{-9}$ & $(2.53 \pm 0.02) \times 10^{-9}$ & $(2.59 \pm 0.02) \times 10^{-9}$ & $(2.45 \pm 0.01) \times 10^{-9}$ \\
$8.91 \times 10^{4}-1.58 \times 10^{5}$ & $1.13 \times 10^{5}$ & $(5.34 \pm 0.05) \times 10^{-10}$ & $(5.86 \pm 0.05) \times 10^{-10}$ & $(6.09 \pm 0.06) \times 10^{-10}$ & $(5.50 \pm 0.04) \times 10^{-10}$ \\
$1.58 \times 10^{5}-2.82 \times 10^{5}$ & $2.04 \times 10^{5}$ & $(1.13 \pm 0.01) \times 10^{-10}$ & $(1.28 \pm 0.01) \times 10^{-10}$ & $(1.31 \pm 0.01) \times 10^{-10}$ & $(1.19 \pm 0.01) \times 10^{-10}$ \\
\hline \hline
\end{tabular}




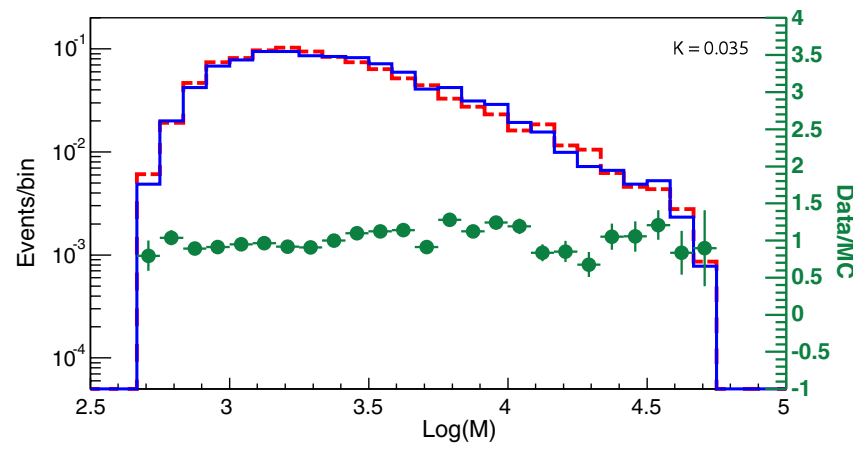

FIG. 5 (color online). Normalized strip multiplicity distribution for Monte Carlo events (dashed red line) and data (solid blue line). The green points represent the ratio of data over Monte Carlo distributions.

A conservative estimate of the uncertainty due to the effect of different run conditions and to seasonal effects has been obtained by analyzing the three runs separately (see Table I), and it turns out to be about $\pm 4 \%$, slightly larger in the edge bins.

(iii) Effects related to the fraction of helium component used to evaluate the Bayesian probabilities. The effect of the variation of the helium component in the range $7 \div 11 \%$ has been evaluated as negligible. In order to evaluate the effect of a large variation of the helium component, the unfolding procedure has been performed again by using a Monte Carlo sample composed of 50\% protons and $50 \%$ helium nuclei. The values of the energy spectrum obtained by using this sample are consistent, within a few percent, with those obtained with the sample containing $9 \%$ of helium.

Therefore we estimate that our result is affected by a total systematic uncertainty not exceeding $10 \%$. In order to have an evaluation of the systematics due to the use of different hadronic interaction models, the conditioned probabilities
$P(M \mid E)$ have been computed for three energy values, namely, $10 \mathrm{TeV}, 30 \mathrm{TeV}, 120 \mathrm{TeV}$, by using also the SIBYLL model $[38,39]$. The results are reported in Fig. 6 as a function of the multiplicity $M$ of the detected showers and compared with the same results obtained by using QGSJET-II. The plots show that the probabilities provided by the two interaction models are very similar, suggesting therefore a very small contribution to the systematic uncertainty.

\section{The light-component spectrum}

The values of the energy spectrum obtained by applying the Bayesian unfolding procedure to all the data collected in the three runs are shown in Fig. 7. The spectrum covers the energy region from 5 up to $200 \mathrm{TeV}$. The measured intensities are reported with the total uncertainty obtained by adding the systematic uncertainties, as estimated in the previous section, to the statistical errors. It can be noted that the total uncertainty on each experimental point does not exceed $10 \%$. As shown in Sec. V, the contribution to the energy spectrum of elements heavier than helium is negligible. Our data are compared with the recent results of the CREAM experiment and with the best fit provided by Hörandel to proton and helium experimental fluxes [3]. The point at $80 \mathrm{TeV}$ represents the " $p+\mathrm{He}$ " intensity measured by EAS-TOP and MACRO experiments [40] at the Gran Sasso Laboratory combining the simultaneous detections of the electromagnetic and Cherenkov light components of atmospheric showers and of high-energy muons. The ARGO-YBJ data agree remarkably well (within about $15 \%$ ) with the values obtained by adding up the proton and helium fluxes measured by CREAM, both concerning the total intensities and the spectrum slope. The value of the spectral index of the power-law fit representing the ARGO-YBJ data is $-2.61 \pm 0.04$ (which should be compared with $\gamma_{\text {proton }}=-2.66 \pm 0.02$ and $\gamma_{\text {helium }}=-2.58 \pm 0.02 \mathrm{ob}-$ tained by CREAM).
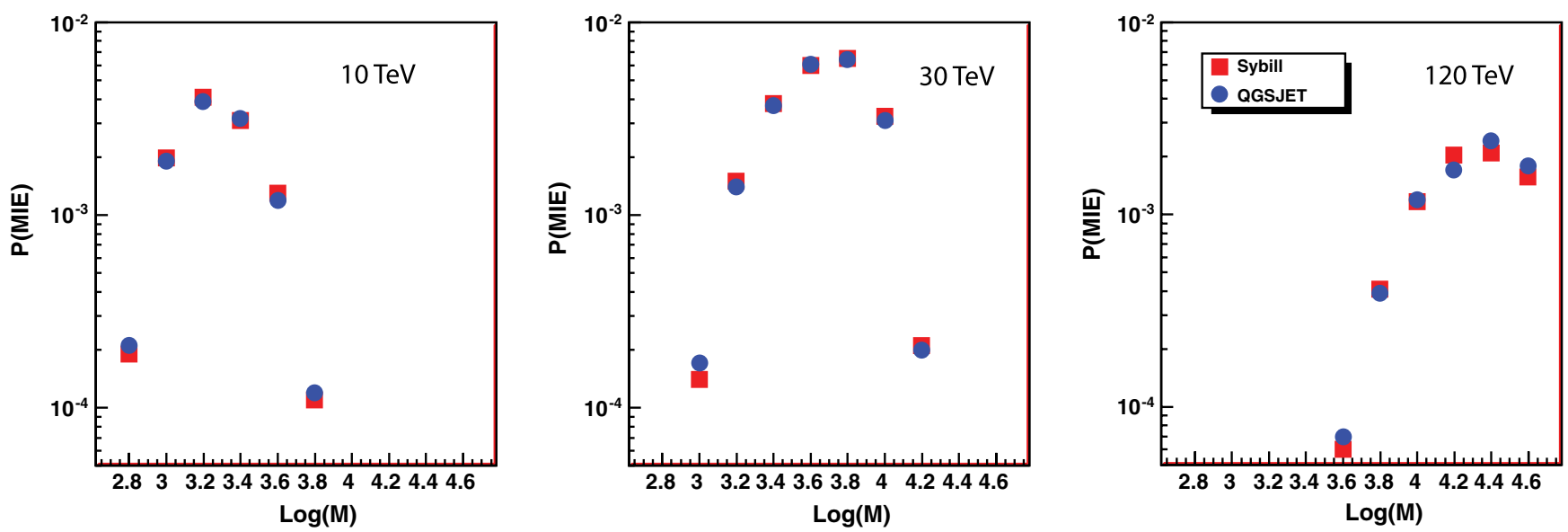

FIG. 6 (color online). Conditioned probability $P(M \mid E)$ for three different energy values (10 TeV, $30 \mathrm{TeV}, 120 \mathrm{TeV})$ by using QGSJET-II (blue circles) and SYBILL (red squares) interaction models. 


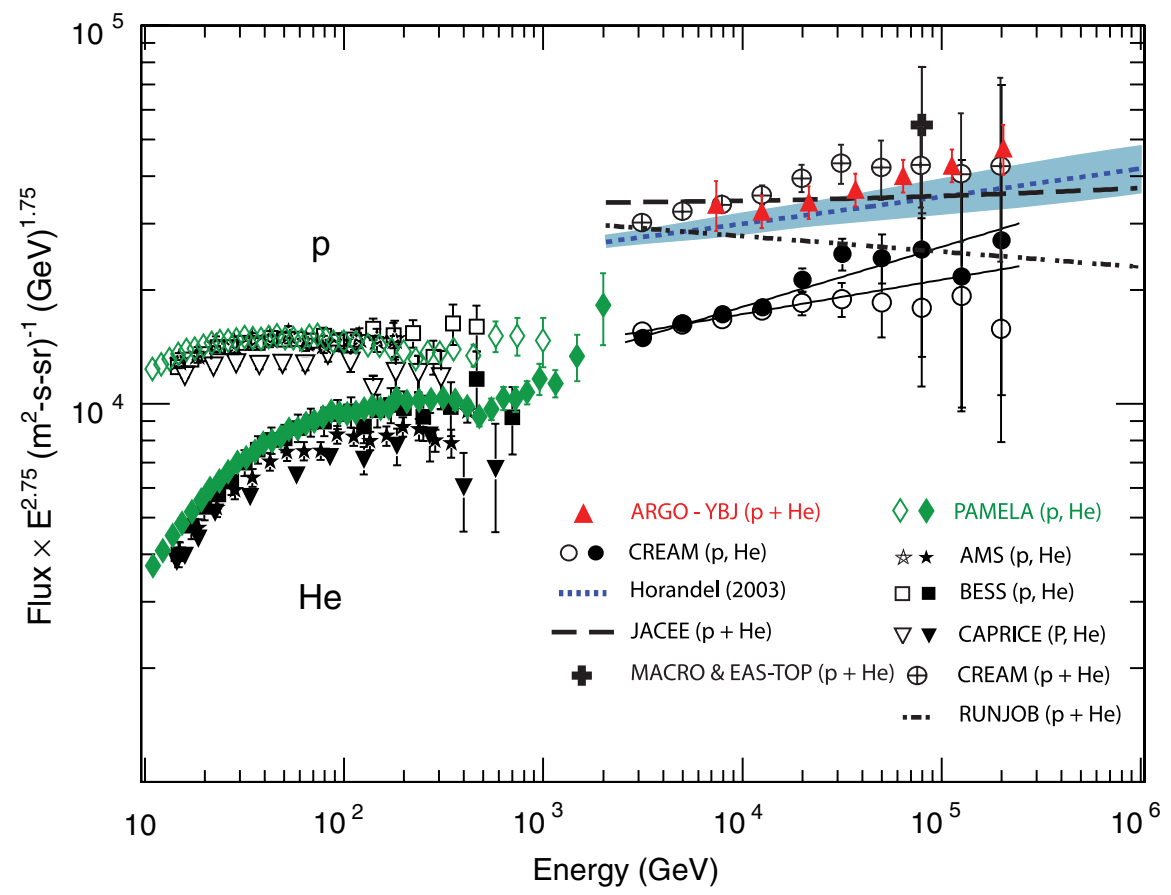

FIG. 7 (color online). The differential energy spectrum of the light component (proton and helium) measured by ARGO-YBJ (filled red triangles) compared with the proton (open circles) and helium spectra (filled circles) measured by the CREAM experiment [43]. The crossed circles represent the sum of the proton and helium data measured by CREAM [5]. The blue dotted line represents the best fit to proton and helium data quoted by Hörandel [3], and the shaded area is obtained considering the errors on the fit parameters. The dashed line represents the JACEE proton and helium measurements [6], and the dash-dotted line represents the RUNJOB proton and helium measurements [30] (the errors on both are not reported). The black cross represents the proton and helium flux measured by EAS-TOP and MACRO Collaborations [40]. The spectra obtained at lower energies by PAMELA (green diamonds) [44], AMS (stars) [45], BESS (squares) [46], and CAPRICE (inverted triangles) [47] are also shown.

\section{DISCUSSION}

The energy spectrum of the light component of the primary cosmic rays from $10^{12}$ to $10^{15} \mathrm{eV}$ plays an important role to understand the mechanisms of acceleration, propagation, and galactic confinement. Spectral differences between the proton and helium components might be related to different types of sources and acceleration environments $[8,41]$. The present analysis does not allow the determination of the individual proton and helium contributions to the measured flux, and shows a discrepancy with the spectra obtained at lower energies from direct measurements by the passive balloon-borne experiments JACEE and RUNJOB. The disagreement with the spectrum obtained adding the proton and helium components quoted by RUNJOB [30] is remarkable. This discrepancy takes place also at energies around $100 \mathrm{TeV}$ where the JACEE and RUNJOB experiments report a proton spectral index of about $-2.8[6,30]$. Deriving the primary energy spectrum from ground-based EAS measurements introduces uncertainties related to the hadronic interaction model underlying the analysis. The strip multiplicity spectrum measured by ARGO-YBJ is mainly due to the electromagnetic component of the shower, the fraction of muons and hadrons being less than $10 \%$. This component is sensitive to parameters governing the longitudinal development of the shower, like the proton-air inelastic cross section and the energy transferred to the leading particle (elasticity). These processes influence the number of particles observed at ground level. A different longitudinal development of the shower could stretch or compress the energy scale in such a way as to affect the spectral shape. In this context it is worthwhile to note that the proton-air inelastic cross section measured by the ARGO-YBJ experiment in the energy range $(1 \div$ 100) $\mathrm{TeV}$ [37] has been found in good agreement with the values set in the CORSIKA/QGSJET code. According to the results shown in Fig. 6, the QGSJET and SYBILL models provide the same description of the longitudinal development of the shower in the energy range of interest. Moreover, dedicated calculations find that the influence on the shower size of different low-energy models (GHEISHA and FLUKA) is negligible [42]. These results give support to the present interpretation of the ARGO-YBJ experimental data.

\section{CONCLUSIONS}

The peculiar features of the ARGO-YBJ experimenthigh segmentation coupled to a digital readout, full 
coverage, and high altitude location-allow the imaging of the front of small size showers induced by primaries with energies down to a few $\mathrm{TeV}$, so far accessible only with balloon-borne experiments. Showers recorded by ARGOYBJ with a number of fired strips in the multiplicity interval $500 \div 50000$ are mainly induced by primaries in the energy range $(1 \div 300) \mathrm{TeV}$. Requiring quasivertical showers $\left(\vartheta<30^{\circ}\right)$ and applying a selection criterion based on the particle density, a sample of events mainly induced by protons and helium nuclei with core inside a fiducial area (of about $40 \times 40 \mathrm{~m}^{2}$ ) has been selected. The contamination by heavier nuclei is found negligible. An unfolding technique based on the Bayesian approach has been applied to the strip multiplicity distribution in order to obtain the differential energy spectrum of the light component (protons and helium nuclei) in the energy range $(5 \div 200) \mathrm{TeV}$. The main uncertainty affecting this analysis is due to systematic effects which do not exceed $10 \%$. Whereas fairly consistent with the recent results of the CREAM experiment, the ARGO-YBJ data indicate a flux of the light component higher with respect to RUNJOB. This measurement bridges the energy gap between the lower energy direct observation and the ground-based EAS experiments.

\section{ACKNOWLEDGMENTS}

This work is supported in China by NSFC (Contract No. 101201307940), the Chinese Ministry of Science and Technology, the Chinese Academy of Science, and the Key Laboratory of Particle Astrophysics, CAS, and in Italy by the Istituto Nazionale di Fisica Nucleare (INFN), and Ministero dell'Istruzione, dell'Università e della Ricerca (MIUR). We also acknowledge the essential support of W. Y. Chen, G. Yang, X. F. Yuan, C. Y. Zhao, R. Assiro, B. Biondo, S. Bricola, F. Budano, A. Corvaglia, B. D'Aquino, R. Esposito, A. Innocente, A. Mangano, E. Pastori, C. Pinto, E. Reali, F. Taurino, and A. Zerbini, in the installation, debugging, and maintenance of the detector.
[1] J. Hörandel, Nucl. Instrum. Methods Phys. Res., Sect. A 588, 181 (2008).

[2] J. Blümer, R. Engel, and J. Hörandel, Prog. Part. Nucl. Phys. 63, 293 (2009).

[3] J. R. Hörandel, Astropart. Phys. 19, 193 (2003).

[4] H. S. Ahn et al., Astrophys. J. 707, 593 (2009).

[5] Y.S. Yoon et al., Astrophys. J. 728, 122 (2011).

[6] K. Asakimori et al., Astrophys. J. 502, 278 (1998).

[7] V. A. Derbina et al., Astrophys. J. 628, L41 (2005).

[8] P. L. Biermann, Astron. Astrophys. 271, 649 (1993).

[9] T. Stanev, P. L. Biermann, and T.K. Gaisser, Astron. Astrophys. 274, 902 (1993).

[10] C. Bacci et al., Nucl. Phys. B, Proc. Suppl. 78, 38 (1999).

[11] C. Bacci et al., Astropart. Phys. 17, 151 (2002).

[12] G. Aielli et al., Nucl. Instrum. Methods Phys. Res., Sect. A 562, 92 (2006).

[13] S. Bussino, E. De Marinis, and S. M. Mari, Astropart. Phys. 22, 81 (2004).

[14] G. Aielli et al., Nucl. Instrum. Methods Phys. Res., Sect. A 608, 246 (2009).

[15] P. Creti et al., for the Argo-YBJ Collaboration, in Proceedings of 29th International Cosmic Rays Conference (Tata Institute of Fundamental Research, Pune, India, 2005)

[16] G. D’Ali Staiti, Nucl. Instrum. Methods Phys. Res., Sect. A 588, 7 (2008).

[17] B. Bartoli et al., Phys. Rev. D 84, 022003 (2011).

[18] A. Aloisio, P. Branchini, A. Budano, S. Catalanotti, P. Creti, F. Galeazzi, G. Marsella, S. Mastroianni, F. Ruggieri, and C. Stanescu, Nucl. Instrum. Methods Phys. Res., Sect. A 568, 847 (2006).

[19] V. Blobel, report, CERN School of Computing, Aiuguablava, Catalonia, Spain, 1984.
[20] G. D’Agostini, Nucl. Instrum. Methods Phys. Res., Sect. A 362, 487 (1995).

[21] D. Heck, J. Knapp, J. Capdevielle, G. Schatz, and T. Thouw, Report No. FZKA 6019, Forschungszentrum Karlsruhe-Wissenhaltliche Berichte, 1998.

[22] W. R. Nelson et al., SLAC Report No. 265, 1985.

[23] A. F. Belayev et al., SLAC Report No. 64991994.

[24] J. Knapp et al., Report No. FZKA 5828, Forschungszentrum Karlsruhe-Wissenhaltliche Berichte, 1996.

[25] N. N. Kalmykov and S. S. Ostapchenko, Phys. At. Nucl. 56, 346 (1993).

[26] H. Fesefeldt, Report No. PITHA 85-02, RWTH Aachen, 1985.

[27] CERN Application Software Group, "GEANT-Detector Description and Simulation Tool," Tech. Rep., CERN Program Library, long write-up, Report No. W5013, 1993.

[28] M. Amenomori et al., Astrophys. J. 678, 1165 (2008).

[29] M. Ave, P. J. Boyle, F. Gahbauer, C. Höppner, J. R. Hörandel, M. Ichimura, D. Müller, and A. Romero-Wolf, Astrophys. J. 678, 262 (2008).

[30] A. V. Apanasenko et al., Astropart. Phys. 16, 13 (2001).

[31] A. Aloisio, P. Branchini, S. Catalanotti, S. Cavaliere, P. Creti, G. Marsella, S. Mastroianni, and P. Parascandolo, IEEE Trans. Nucl. Sci. 51, 1835 (2004).

[32] G. Aielli et al., Astropart. Phys. 30, 287 (2009).

[33] J. Nishimura, in Prog. Cosmic Ray Physics (North Holland, Amsterdam 1956).

[34] K. Kamata and J. Nishimura, Prog. Theor. Phys. Suppl. 6, 93 (1958).

[35] K. Greisen, in Prog. Cosmic Ray Physics (North Holland, Amsterdam, 1956).

[36] G. D’ Agostini, arXiv:1010.0632.

[37] G. Aielli et al., Phys. Rev. D 80, 092004 (2009). 
[38] R. S. Fletcher, T. K. Gaisser, P. Lipari, and T. Stanev, Phys. Rev. D 50, 5710 (1994).

[39] J. Engel, T. K. Gaisser, P. Lipari, and T. Stanev, Phys. Rev. D 46, 5013 (1992).

[40] M. Aglietta et al., Astropart. Phys. 21, 223 (2004).

[41] Y.M. Butt and A.M. Bykov, Astrophys. J. 677, L21 (2008).
[42] H. Ulrich et al., Nucl. Phys. B, Proc. Suppl. 175-176, 273 (2008).

[43] H. S. Ahn et al., Astrophys. J. Lett. 714, L89 (2010).

[44] O. Adriani et al., Science 332, 69 (2011).

[45] J. Alcaraz et al., Phys. Lett. B 494, 193 (2000).

[46] S. Haino et al., Phys. Lett. B 594, 35 (2004).

[47] M. Boezio et al., Astropart. Phys. 19, 583 (2003). 